\title{
COMPOSIÇÃO DA MACROFAUNA BENTÔNICA DURANTE O PROCESSO DE RECUPERAÇÃO DA MATA CILIAR DO RIO MANDU EM POUSO ALEGRE, MG.
}

\author{
Marcos Vinícius Nunes ${ }^{1}$ \\ Emerson Ribeiro Machado ${ }^{2}$ \\ José Valdecir de Lucca ${ }^{3}$ \\ Odete Rocha ${ }^{4}$
}

\section{RESUMO}

Os macroinvertebrados bentônicos têm gradativamente recebido maior atenção devido à sua importância nos processos ecológicos, por meio de sua participação no fluxo de energia e na ciclagem de nutrientes nos sistemas aquáticos, desempenhando papel importante na troca de fósforo e nitrogênio entre o sedimento e a água de interface. Além disso, eles participam das cadeias alimentares em várias comunidades aquáticas, especialmente como alimento para peixes e aves aquáticas. $O$ presente trabalho foi realizado em um trecho degradado do rio Mandu, em Pouso Alegre, MG, e teve como objetivo principal analisar a influência da recomposição mata ciliar sobre a comunidade de macroinvertebrados bentônicos neste rio. A coleta dos macroinvertebrados foi realizada

\footnotetext{
${ }^{1}$ Programa de Pós-Graduação em Ecologia e Recursos Naturais (PPG-ERN)/ Universidade Federal de São Carlos (UFSCAr)

${ }^{2}$ Universidade do Vale do Sapucaí/Departamento de Ciências Biológicas, Pouso Alegre, MG

${ }^{3}$ Universidade Federal de São Carlos/Departamento de Ecologia e Biologia Evolutiva, São Carlos SP

${ }^{4}$ Universidade Federal de São Carlos/Departamento de Ecologia e Biologia Evolutiva, São Carlos SP
} 
com amostrador Surber em dezembro de 2007 e dezembro de 2008. Após a coleta 0 material foi triado e identificado ao nível de família com o auxilio de bibliografia especializada. Houve um aumento significativo da abundância após o processo de recuperação da mata ciliar. Foram coletados nas duas campanhas um total de 1671 espécimes, sendo que destes 95 foram coletados em dezembro de 2007 e 1576 em dezembro de 2008.

Palavras-chave: Recursos hídricos; mata ciliar; macroinvertebrados bentônicos.

\section{INTRODUÇÃO}

As mudanças em ambientes aquáticos podem ser visualmente notadas quando se observam as comunidades que vivem nestes ecossistemas. Dentre todas as comunidades encontradas nos sistemas de águas doces a comunidade de macroinvertebrados bentônicos é frequentemente utilizada na avaliação de impactos ambientais e no monitoramento biológico (Egler, 2002; Goulart e Callisto, 2003; Buss 2004). Os macroinvertebrados bentônicos são organismos que habitam os sedimentos dos ecossistemas aquáticos continentais, que podem ser os fundos de riachos, corredeiras, rios, represas e lagos, durante parte do seu ciclo de vida ou toda a vida associados aos mais diversos tipos de substratos, tanto orgânicos (folhiço, macrófitas aquáticas, etc.), quanto inorgânicos (cascalho, areia, rochas, etc.). Geralmente possuem tamanho de corpo superior a $0,2 \mathrm{~mm}$, sendo possível a observação a olho nu e ficam retidos em malhas de rede entre 0,2 e 0,5 mm de abertura de malha (Rosenberg e Resh, 1993; Callisto 2004; Silveira et al, 2004; Pinho et al, 2006). Os macroinvertebrados bentônicos têm sua distribuição controlada por vários fatores, dentre eles destacando-se a disponibilidade e qualidade do alimento, o tipo de sedimento ou substrato, a temperatura do meio, morfologia do ecossistema, presença de vegetação aquática, presença e extensão de mata ciliar e mudanças na produtividade primária. (Ward et al., 1995; Esteves, 1998; Galdean et al., 2000). A comunidade de macroinvertebrados bentônicos pode apresentar grande diversidade de espécies e diversas formas e modos 
de vida adaptando-se aos habitats locais. O componente de maior evidência entre os grupos de macroinvertebrados bentônicos são geralmente os Insecta, com destaque para as larvas de insetos, na sua forma larvar, geralmente encontradas em grande quantidade, no caso dos grupos Diptera aquáticos, Ephemeroptera, Plecoptera, Odonata, Trichoptera e outros grupos de invertebrados como os Anellida e Mollusca entre outros (Vieira et al, 1998; Mandaville, 2002; Buss, 2004; Silveira, 2004; Moreno e Callisto, 2005; Lucca, 2006). A comunidade bentônica exerce grande influência na dinâmica dos ecossistemas aquáticos por atuarem no processo de biorrevolvimento, onde o sedimento é escavado pela atividade dos macroinvertebrados bentônicos. $O$ biorrevolvimento depende basicamente de quatro fatores principais: tamanho do organismo, densidade, atividade e capacidade de penetração no sedimento (Berg et. al., 1997; Esteves, 1998; Beier e Traunspurger, 2001; Mandaville, 2002; Lucca, 2006). O objetivo deste trabalho foi comparar as mudanças ocorridas na comunidade de macroinvertebrados bentônicos após um ano do início do processo de recuperação de um trecho da mata ciliar do rio Mandu em Pouso Alegre-MG, Brasil.

\section{MATERIAL E MÉTODOS}

A área em estudo e que foi reflorestada localiza-se em um trecho do rio Mandu que passa pelo bairro rural Cajuru em Pouso Alegre-MG. Foram selecionados quatro (4) pontos amostrais para serem realizadas as coletas dos macroinvertebrados bentônicos. $A$ coleta foi realizada nos meses de dezembro de 2007 e dezembro de 2008. Para a coleta dos macroinvertebrados utilizou-se um amostrador tipo Surber, que consiste de um delimitador de área de $0,9 \mathrm{~m}^{2}$ associado a uma rede de malha de 250 micra de abertura. As coletas foram realizadas na zona litorânea, em duplicata, e seguiu o Protocolo de coleta e preparação de amostras segundo Silveira, 2004 e Brandimarte et al. 2004. As amostras foram acondicionadas em sacos plásticos com água do local, etiquetadas e transportadas para o Laboratório de Ecologia da UNIVAS (Universidade do Vale do Sapucaí). No laboratório as amostras foram lavadas em água corrente sobre peneiras com abertura de malhas de 1,00 e $0,25 \mathrm{~mm}$. Os organismos foram triados em bandeja transiluminada e preservados em álcool $70 \%$ até a identificação. Com o auxílio de um estereomicroscópio os exemplares foram isolados e identificados taxonomicamente até o 
nível de família por meio de chaves taxonômicas especializadas como: Pérez (1988), Trivinho-Strixino e Strixino (1995), Merritt e Cummins (1996) e Costa (2006). Após a identificação os organismos foram fixados em nova solução de álcool $70 \%$. Para avaliar a estrutura da comunidade de macroinvertebrados bentônicos foi calculada a frequência de ocorrência (\% de indivíduos), a riqueza taxonômica (através do número total de táxons encontrados em cada ponto amostral), o número de indivíduos coletados e a densidade (calculada considerando-se o número total de indivíduos e a área do amostrador).

\section{RESULTADOS}

Foram coletados no total 1671 organismos, sendo desde 95 organismos coletados na coleta de dezembro de 2007 e 1576 na coleta de dezembro de 2008 (Figura 1). Foram registrados 25 táxons diferentes, com ocorrência de 15 táxons em dezembro de 2007 e 23 em dezembro de 2008. (Figura 2). Os Chironomidae foram os mais representativos nas 2 campanhas $(64,69 \%$ da fauna total) (Tabela 1). Em dezembro de 2007 este grupo representou $47,37 \%$ do total de macro-invertebrados e em 2008, 65,74\% (Tabela 1). Na Figura 3 é mostrada a riqueza de espécies registrada nas 2 campanhas realizadas. $\mathrm{Na}$ segunda campanha (dezembro de 2008) houve um aumento marcante da riqueza taxonômica (Figura 3) e da densidade (Figura 4) em todos os pontos de amostragem.

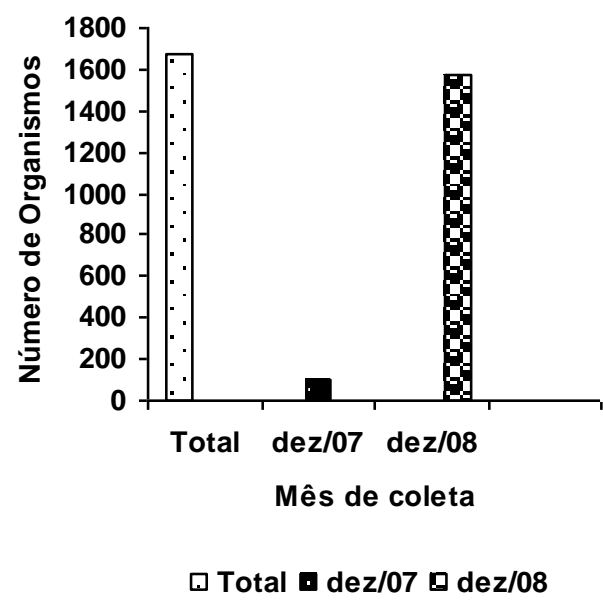

Figura 1- Número de organismos coletados nas campanhas de dezembro de 2007 e dezembro de 2008 e o total. 


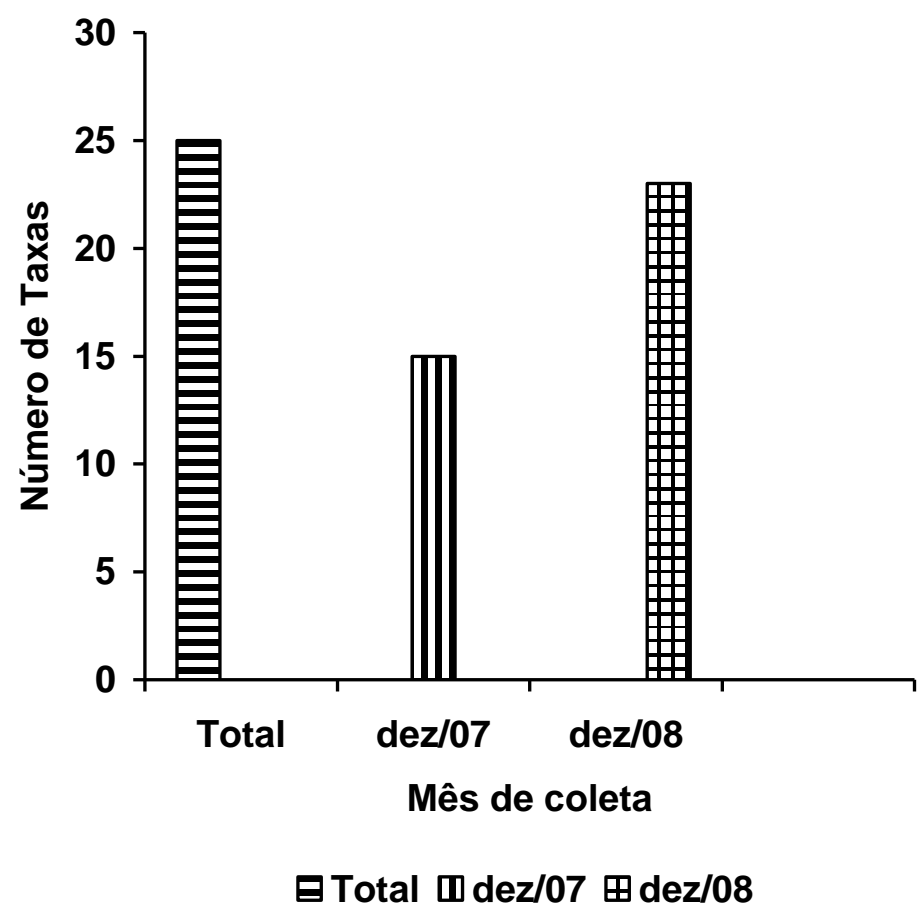

Figura 2 - Riqueza de táxons dos macroinvertebrados bentônicos registrada nas campanhas de dezembro de 2007 e 2008.

Tabela 1- Freqüência de ocorrência dos macroinvertebrados bentônicos registrada nas campanhas de dezembro de 2007 e 2008.

\begin{tabular}{|c|c|c|c|}
\hline Táxons & Dez/07 & Dez/08 & Total \\
\hline Ceratopogonidae & $\nabla$ & $\nabla$ & $\nabla$ \\
\hline Chironomidae & - & $\bullet$ & $\bullet$ \\
\hline Culicidae & & $\nabla$ & $\nabla$ \\
\hline Simuliidae & $\nabla$ & $\nabla$ & $\nabla$ \\
\hline Baetidae & - & $\boldsymbol{\nabla}$ & $\nabla$ \\
\hline Leptophlebiidae & $\boldsymbol{\nabla}$ & $\nabla$ & $\nabla$ \\
\hline Tricorythidae & & $\boldsymbol{\nabla}$ & $\boldsymbol{\nabla}$ \\
\hline Perlidae & & $\nabla$ & $\nabla$ \\
\hline Calamoceratidae & $\nabla$ & $\nabla$ & $\nabla$ \\
\hline Hydrobiosidae & $\nabla$ & $\boldsymbol{\nabla}$ & $\boldsymbol{\nabla}$ \\
\hline Hydropsychidae & & $\boldsymbol{\nabla}$ & $\boldsymbol{\nabla}$ \\
\hline Philopotamidae & & $\nabla$ & $\nabla$ \\
\hline Leptoceridae & $\nabla$ & $\nabla$ & $\nabla$ \\
\hline Calopterygidae & $\boldsymbol{\nabla}$ & & $\boldsymbol{\nabla}$ \\
\hline
\end{tabular}



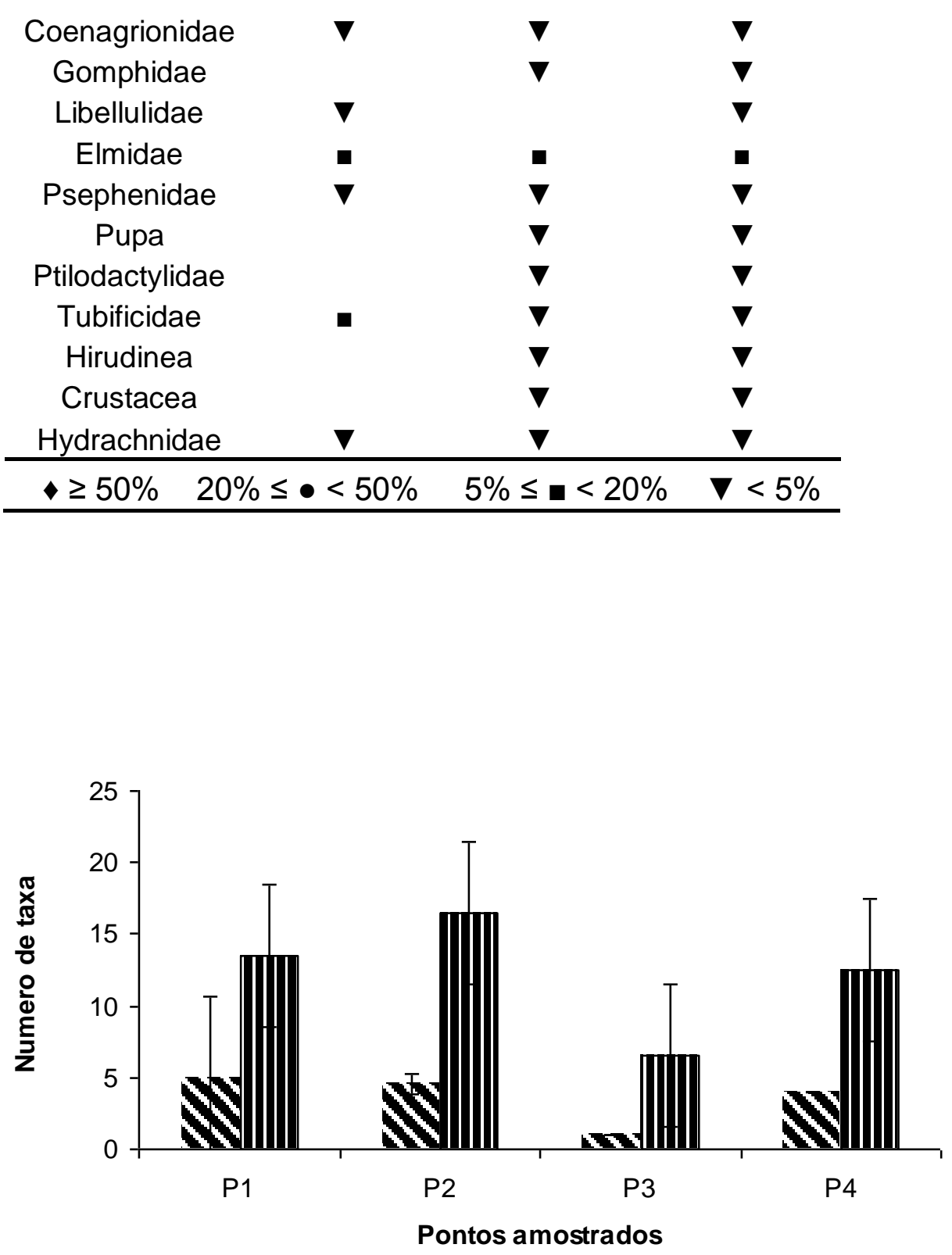

v Dezembro 2007 n Dezembro 2008

Figura 3 - Riqueza de táxons dos macroinvertebrados bentônicos registrada nos 4 pontos de amostragem nas campanhas de dezembro de 2007 e 2008. 


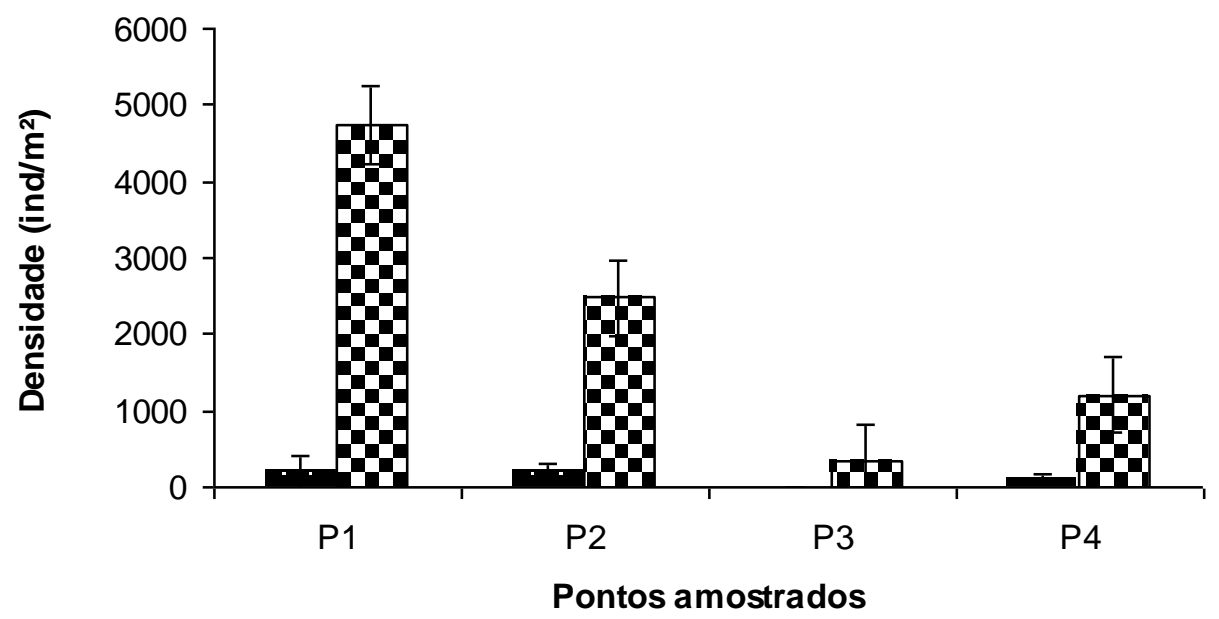

a Dezembro 2007 Dezembro 2008

Figura 4 - Densidade dos macroinvertebrados bentônicos registrado nos 4 pontos de amostragem nas campanhas de dezembro de 2007 e 2008.

\section{DISCUSSÃO}

O aumento na riqueza taxonômica e na densidade dos macroinvertebrados bentônicos entre a campanha de dezembro de 2007 e dezembro de 2008, provavelmente está relacionado com a recuperação da mata ciliar no trecho em estudo. Alguns autores como os de Corbi (2006), Paula \& Fonseca-Gessner (2008), Guereschi (2004) verificaram maiores valores de riqueza em áreas em recomposição e com cobertura florestal, ao contrário de áreas que não apresentam mata ciliar. Isto deve ao fato da mata ciliar proporcionar maior disponibilidade de alimento e habitats (Hynes, 1970). A elevada abundância de Chironomidae $(64,69 \%)$ e a sua ocorrência em todos os pontos de amostragem relaciona-se também ao fato de que os Chironomidae estão amplamente distribuídos, ocorrendo tanto em ambientes lóticos como lênticos e geralmente em elevadas densidades (Pérez, 1988 e Costa 2006). 


\section{CONCLUSÔES}

- Houve aumento da riqueza e densidade dos macroinvertebrados bentônicos entre dezembro de 2007 e 2008;período de recomposição da mata ciliar;

- Os Chironomidae foram os macroinvertebrados bentônicos mais representativos nos dois períodos amostrados confirmando a importância deste grupo para as comunidades de macroinvertebrados em águas doces tropicais;

- A preservação e recuperação da mata ciliar aparentemente refletiram de forma positiva para o aumento da riqueza de espécies na comunidade de macroinvertebrados bentônicos. 


\section{Referências}

Beier S.; Traunspurger, W. 2001. The meiofauna community of two small German streams as indicator of pollution. Journal of Aquatic Ecosystem Stress and Recovery n.8.

Berg, M. S.; Coops, H.; Noordhuis, R.; Schie, J.; Simons, J. 1997. Macroinvertebrate communities in relation to submerged vegetation in two Chara-dominated lakes. Kluwer Academic Publishers. Printed in Belgium, Amsterdam.

Brandimarte, A. L.; Shimizu, G. Y.; Anaya, M.; Kuhlmann, M. L. Amostragem de invertebrados bentônicos. In: Bicudo, C. E. M.; Bicudo, D. C. 2004. Amostragem em Limnologia. São Carlos, Rima.

Buss, D. F. 2004. Importância do biomonitoramento e, programas de análise da qualidade da água de rios. Disponível em: http://www.bioletim.hpg.ig.com.br/llI 3/artigos/buss.htm. Acesso em 30/05/2008.

Callisto, M. 2004. Bioindicadores de qualidade de água. Disponível em: http://www.fundep.ufmg.br/homepage/cases_imprimir.asp?cod=448. Acesso em 12/11/2006.

Corbi, J. J. 2006. Influência de práticas de manejo de solo sobre os macroinvertebrados aquáticos de córregos: ênfase para o cultivo de cana-de-açúcar em áreas adjacentes. Tese de Doutorado-Programa de Pós-graduação em Ecologia e Recursos Naturais do Centro de Ciências Biológicas e da Saúde, Universidade Federal de São Carlos, São Carlos.

Costa, C. (ed). 2006. Insetos Imaturos. Metamorfose e Identificação. Ribeirão Preto: Holos.

Egler, M. 2002. Utilizando a Comunidade de Macroinvertebrados Bentônicos na Avaliação da Degradação Ambiental de Ecossistemas de Rios em Áreas Agrícolas. RJ, Brasil. Dissertação de Mestrado-Programa de Pós-graduação da Escola Nacional de Saúde Pública da Fundação Oswaldo Cruz. Rio de Janeiro.

Esteves, F. A. 1998. Fundamentos de limnologia. Rio de Janeiro, 2 ed., Interciência.

Galdean, N.; Callisto, M.; Barbosa, F.A.R. 2000. Lotic Ecosystems of Serra do Cipó, southeast Brazil: water quality and a tentative classification based on the benthic macroinvertebrate community. Aquatic Ecosystem Health and Management.

Goulart, M.; Callisto, M. 2003. Bioindicadores de qualidade de água como ferramenta em estudos de impacto ambiental. Revista da FAPAM, ano 2, n.1.

Guereschi, R. M. 2004. Macroinvertebrados bentônicos em córregos da Estação Ecológica de Jataí, Luiz Antônio, SP: subsídios para o monitoramento ambiental. Tese de Doutorado-Programa de Pós-graduação em Ecologia e Recursos Naturais do Centro de Ciências Biológicas e da Saúde, Universidade Federal de São Carlos, São Carlos. 
Hynes, H. B. N. 1970. The ecology of running waters. Toronto Press, Toronto.

Lucca, J. V. 2006. Caracterização Limnológica e Análise de Comunidades Bentônicas Sujeitas à Invasão por Espécies exóticas, em lagos do Vale do Rio Doce, MG, Brasil. Tese de Doutorado-Programa de Pós-graduação em Ciências da Engenharia Ambiental da Escola de Engenharia de São Carlos, Universidade de São Paulo, São Carlos.

Mandaville, S.M. 2002. Benthic Macroinvertebrates in Freshwaters-Taxa Tolerance Values, Metrics, and Protocols. In: Protocols for Measuring Biodiversity: Benthic Macroinvertebrates in Fresh Waters- EMAN (Ecological Monitoring and Assessment Network).

Merrit, R.; Cummins, K. 1996. An introduction to the aquatic insects of North America. 3 ed. Hunt Publishing, Kendall.

Moreno, P.; Callisto, M. 2005. Indicadores biológicos: a vida na lama. Ciência Hoje, vol. 36, n. 213.

Paula, M. C. e Fonseca-Gessner, A. A. 2008. Macroinvertebrados bentônicos em córregos de baixa ordem em dois fragmentos de mata ciliar com diferentes estados de conservação, no Estado de São Paulo. UFSCar, São Carlos.

Pérez, G. A. R. 1988. Guía para el estudio de los macroinvertebrados acuáticos del Departamento de Antioquia. Bogotá, Fondo Fen Colômbia, Colciencias/Universidad de Antioquia.

Pinho, L. at al. 2006. Tópicos especiais: macroinvertebrados bentônicos. In: Boletim da Sociedade Brasileira de Limnologia. Ano 2, no 35.

Rosemberg, D. M.; Resh, V. H. (eds). 1993. Freshwater biomonitoring and benthic macroinvertebrates. Chappman \& Hall, New York, NY.

Silveira, M. P. et all. 2004. Protocolo de coleta e preparação de amostras de macroinvertebrados bentônicos em riachos. SP, 7p. Comunicado técnico. Embrapa Meio Ambiente, Jaguariúna, SP.

Trivinho-Strixino, S.; Strixino. G. 1995. Larvas de Chironomidae (Diptera do Estado de São Paulo: guia de identificação de diagnose dos gêneros. São Carlos, Programa de Pós-Graduação em Ecologia e Recursos Naturais, Universidade Federal de São Carlos.

Ward, D.; Holmes, N.; José, P. 1995. The New Rivers \& Wildlife Handbook. RSPP, NRA e The Wildlife Trusts, Bedfordshire. 